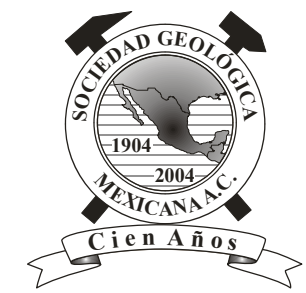

\title{
Caracterización geoquímica y geoeléctrica de un sitio contaminado por hidrocarburos en el estado de Puebla, México
}

\author{
Alejandrina Castro ${ }^{1, *}$, Diana Acevedo-Berruecos ${ }^{1}$, Javier Urbieta $^{1}$, Rosario Iturbe ${ }^{1}$, \\ Omar Delgado-Rodríguez ${ }^{2}$ \\ ${ }^{1}$ Instituto de Ingeniería, Universidad Nacional Autónoma de México, Ciudad Universitaria, 04510, México D.F. \\ ${ }^{2}$ Instituto Mexicano del Petróleo, Eje Central Lázaro Cárdenas Norte 152, San Bartolo Atepehuacan, Gustavo A. Madero, 07730, \\ México D.F. \\ *aacr@pumas.ii.unam.mx
}

\begin{abstract}
Resumen
Dos métodos son utilizados para caracterizar un sitio contaminado con hidrocarburos: muestreo de suelo y análisis químico como método directo y tomografía de resistividad eléctrica (ERT, por sus siglas en inglés) como método indirecto. La caracterización con el método directo se basó en la NOM-138-SEMARNAT/SS-2003, donde se obtuvieron 42 muestras, incluidos 4 duplicados, con lo que se detectó que el contaminante principal en el subsuelo es diésel, siendo el volumen de suelo afectado aproximadamente $1301 \mathrm{~m}^{3}$. El método ERT se realizó en dos etapas: seis perfiles en la primera etapa, paralelos entre sí con una distancia entre ellos de 4 m, con un arreglo electródico dipolo-dipolo con una separación entre electrodos de $0.5 \mathrm{~m}$. En la segunda etapa se realizaron dos perfiles ubicados en los extremos noroeste y sureste del sitio utilizando un arreglo Wenner-Schlumberger con una separación entre electrodos de $0.75 \mathrm{~m}$ (cerca de los puntos de muestreo con mayores niveles de contaminación PUE1 y PUE18), cuyo resultado muestra una posible delineación del contaminante, en correspondencia con el análisis granulométrico realizado en las muestras de suelo, el cual indica la existencia de un suelo arenoso de alta permeabilidad. A pesar de que la ubicación y longitud de los perfiles de ERT en ambas etapas estuvieron afectadas por la accesibilidad al sitio, los resultados muestran claramente anomalías de alta resistividad ( $\rho>600$ Ohm-m) asociadas a la presencia de una cobertura resistiva que incluye contaminación reciente por hidrocarburos. La estratigrafía compuesta de sedimentos arenosos propios del sitio manifiesta variaciones de resistividades entre 25 y $150 \mathrm{Ohm}-\mathrm{m}$ debidas a variaciones de humedad. Tanto los resultados del muestreo de suelo y análisis químico, como los de ERT, indican que la contaminación es en general de poca extensión horizontal; sin embargo, dos perfiles de ERT muestran una capa geoeléctrica de mayor extensión, con valores de resistividad inferior a los $10 \mathrm{Ohm}-\mathrm{m}$ como señal evidente de contaminación madura, aunque se requiere de nuevas perforaciones y muestreo de suelo para su confirmación.
\end{abstract}

Palabras clave: caracterización, contaminación puntual, hidrocarburos, tomografía eléctrica.

\section{Resumen}

Two methods are used to characterize a site contaminated with hydrocarbons: soil sampling and chemical analysis as direct methods, and electrical resistivity tomography (ERT) as an indirect method. The application of the direct method was based on the NOM-138SEMARNAT/SS-2003; 42 soil samples were collected, including 4 duplicates, concluding that the main contaminant in the subsoil is diesel and that the volume of affected soil is approximately $1301 \mathrm{~m}^{3}$. The ERT method was carried out in two stages: six profiles in the first stage, parallel to each other, with a distance of $4 \mathrm{~m}$ between them, using a dipole-dipole arrangement with a distance of $0.5 \mathrm{~m}$ between electrodes. In the second stage, two profiles located in the northwest and southeast ends of the site using a Wenner-Schlumberger array with a separation of $0.75 \mathrm{~m}$ between electrodes (near the points PUE1 and PUE18, which are the most polluted)may provide the possible delineation of the contaminant in correspondence to the granulometric analysis performed in soil samples, which indicates 
the existence of a sandy soil of high permeability. Eventhough the location and length of the ERT profiles in both stages were affected by the accesibility of the industrial site, the results clearly show anomalies of high resistivity $(\rho>600$ Ohm- $m$ ) associated with the presence of recent hydrocarbon contamination. Moisture changes in the compound stratigraphy of the sandy sediments cause resistivity variations between 25 and 150 Ohm-m. Both soil sampling and chemical analyses, and the ERT results, indicate that the hydrocarbon contamination has generally low horizontal extension; however, two ERT profiles show a conductive layer of greater extension with resistivity values lower than $10 \mathrm{Ohm}-\mathrm{m}$, indicating the possible presence of mature contamination, eventhough new drilling and soil sampling are required to confirm this assumption.

Keywords: characterization, punctual contamination, hydrocarbons, electrical tomography.

\section{Introducción}

En México existen diferentes fuentes generadoras de contaminación por hidrocarburos. La presencia de tales contaminantes en el suelo tiene repercusiones directas en el medio ambiente, en las especies vegetales, animales $\mathrm{y}$ en la salud humana por efectos del contacto directo, inhalación o ingestión de los mismos. Su transporte a través del suelo puede originar la contaminación de los acuíferos, deteriorando la calidad del agua ahí presente. El problema tratado en este trabajo es la contaminación de suelos por hidrocarburos en un ex taller de ferrocarriles localizado en el estado de Puebla. Ferrocarriles Nacionales de México (FNM) es una de las empresas que ha contribuido a la contaminación del agua y del suelo debido principalmente al inadecuado almacenamiento de residuos y combustibles, tales como la creosota y los aceites gastados (PROFEPA, 2002). Los aspectos de protección ambiental no fueron considerados en FNM de manera formal sino hasta el año 1993, cuando se crea el Comité de Protección Ambiental. En ese mismo año se elaboró un plan maestro para el periodo 1993-1996 que contemplaba la realización de auditorías ambientales en las 56 instalaciones de FNM en todo el territorio nacional (Rocha, 2000). Seis meses después del inicio de este plan comenzó un programa de caracterización y análisis de áreas contaminadas, el cual revela contaminación en el $90 \%$ de las instalaciones debido a hidrocarburos en agua y suelo, principalmente diésel y aceite (Madrigal, 1998).

A la fecha, existen algunos avances en México en materia ambiental relacionada con los hidrocarburos, uno de ellos es la norma oficial mexicana NOM-138-SEMARNAT/ SS-2003, con base en la cual se realizó el muestreo para la caracterización del ex taller de ferrocarriles. La técnica que la norma estipula en la delimitación de áreas contaminadas por hidrocarburos es el muestreo directo, que consiste en la recolección de muestras de suelo las cuales se analizan en el laboratorio para identificar la concentración de hidrocarburos. En el presente trabajo se propone una técnica geofísica como alternativa complementaria al muestreo directo, basada en la medición de la resistividad del suelo impregnado del contaminante mediante la inyección de una corriente eléctrica.
En los últimos años, la resolución de problemas de carácter ambiental con ayuda de la geofísica ha cobrado singular importancia dentro de las ciencias de la Tierra (Volkov et al., 2000; Lopes de Castro y Gomes Castelo Branco, 2003; Shevnin et al., 2005; Delgado-Rodríguez et al., 2006; Hernández Márquez y Rodrígez Villuendas, 2008). La geofísica propone diferentes procedimientos $\mathrm{y}$ aplicaciones por medio de métodos de prospección eléctrica y electromagnética (Pellerin, 2002), como por ejemplo, el método de Tomografía de Resistividad Eléctrica (Shevnin et al., 2005) y el método de Radar de Penetración Terrestre (GPR, por sus siglas en inglés) (Dehaini et al., 2000) entre otros, que son una valiosa herramienta para la evaluación de los problemas ambientales (EPA, 2000). La presencia de contaminantes en las rocas, en particular los hidrocarburos, modifica la resistividad eléctrica, creando un contraste de resistividades entre las formaciones limpias y las contaminadas (Sauck, 2000). Esta diferencia depende, entre otros factores, de la antigüedad del derrame de hidrocarburo. Cuando un diésel presenta algún grado de intemperismo, los compuestos volátiles se pierden en una gran proporción (70 \% en 30 días) (Saval y Lesser, 1999). De esta manera, los compuestos que quedan formando el diésel intemperizado son más difíciles de biodegradar debido a su estabilidad. Esto es muy importante de tomar en cuenta cuando se diseñan las técnicas de remediación de un sitio. Para el caso de derrames recientes, los suelos reportan valores de alta resistividad (debido a la firma resistiva del hidrocarburo) y cuando el derrame es antiguo, se observa lo contrario, es decir, la contaminación presenta valores de baja resistividad (Atekwana et al., 2000). Según Sauck (2000), la anomalía de baja resistividad se debe a un aumento de los sólidos totales disueltos en el entorno ácido creado por la acción bacteriana en la parte inferior de la zona vadosa. Sin embargo, estudios posteriores indican que el principal causante de la anomalía de baja resistividad es la alta conductividad de las biopelículas (conductividad superficial), producidas por la actividad bacteriana en las paredes de los poros del suelo afectado durante el proceso de biodegradación, además de la disminución de los elementos ligeros en los hidrocarburos (Abdel Aal et al., 2004).

Algunos de los trabajos realizados en la caracterización con métodos indirectos en México, fueron realizados 
por Shevnin et al., (2005) en un sitio contaminado por hidrocarburos como resultado de los trabajos de perforación y explotación de un pozo petrolero en el estado de Tabasco. Ellos realizaron la caracterización geoeléctrica utilizando el método de Tomografía de Resistividad Eléctrica (ERT, por sus siglas en inglés), con la obtención de secciones y mapas de resistividad y la configuración de las plumas de contaminación madura en correspondencia con anomalías de baja resistividad. La aportación principal de este trabajo es la definición de la frontera geoeléctrica entre suelo limpio y contaminado mediante un proceso de modelación petrofísica (Shevnin et al., 2005), basado en mediciones de resistividad en muestras de suelo limpio y contaminado colectadas en el sitio.

Un caso más fue estudiado en un sitio afectado por una fuga de gasolina ocurrida en una línea de ducto cerca de Cárdenas, Tabasco (Delgado-Rodríguez et al., 2006). El estudio consistió en la aplicación del método ERT a lo largo de seis perfiles paralelos en el "derecho de vía" de los diferentes ductos. Sus resultados presentados en mapas y secciones muestran que, aunque el grado de contaminación en este sitio fue bajo, se localizaron dos zonas contaminadas dentro del acuífero arenoso. Tanto el acuífero arenoso como el acuitardo arcillo-arenoso fueron caracterizados, no sólo con base en los valores de resistividad, sino en parámetros petrofísicos como contenido de arcilla, porosidad y capacidad de intercambio catiónico. Asimismo, fueron reinterpretados a partir de los valores de resistividad, utilizando un algoritmo de inversión que toma en cuenta la salinidad del agua de poro, siendo este el aspecto más novedoso del trabajo.

El presente trabajo trata sobre el diagnóstico de un sitio industrial contaminado por hidrocarburos, donde estudios geoquímicos en 42 muestras de suelo indicaron que el contaminante presente es diésel, mostrando plumas de contaminación de poca extensión horizontal. El método ERT se aplicó como alternativa complementaria al muestreo directo, mostrando la posibilidad de existencia de contaminación madura y reciente, lo que requiere de perforaciones adicionales para su confirmación.

\section{Descripción del área de estudio}

El área de estudio se encuentra ubicada en la parte central del estado de Puebla, en la ciudad del mismo nombre. El marco geológico-regional del área de estudio fue obtenido a partir de las cartas geológico-mineras Acatlán de Osorio E14-B83, Chiautla E14-B72 y Zacatlán E14-B14 del Servicio Geológico Mexicano (SGM, 2010).

La geología del estado de Puebla está conformada principalmente por rocas sedimentarias e ígneas. Su basamento se encuentra conformado por una secuencia paleozoica indiferenciada altamente plegada, fracturada y foliada. La granulometría característica de la zona de estudio, de acuerdo al análisis granulométrico realizado en las muestras de suelo extraídas, indican que el suelo predominante es arena de fina a gruesa. En la zona de estudio se llevaron a cabo trabajos de nivelación del terreno por parte de la empresa, de manera que, al menos superficialmente, se encontró material de relleno.

La estación meteorológica Puebla es la más representativa de las condiciones de la región estudiada; manifiesta una temperatura promedio de $16^{\circ} \mathrm{C}$. De acuerdo con la misma estación meteorológica, se reporta una precipitación promedio del año más seco (1949) de aproximadamente $38 \mathrm{~mm}$ y el promedio del año más lluvioso (1992) de $108 \mathrm{~mm}$. La región hidrológica que comprende la zona de estudio es la del Río Balsas, cuenca del río Atoyac (INEGI, 2004). El acuífero correspondiente a la ciudad de Puebla es el 2104 Valle de Puebla. Las características geohidrológicas corresponden a un sistema donde se distinguen verticalmente tres acuíferos: superior, medio y profundo. El acuífero superior está formado por la secuencia aluvial y volcánica del Cuaternario, que recibe directamente una recarga subterránea proveniente de las partes altas de la cuenca. Su espesor varía de unos cuantos metros en sus bordes a más de $200 \mathrm{~m}$ en su porción central, que corresponde con el área circundante a la ciudad de Puebla (CONAGUA, 2009). La permeabilidad es de media a alta, el acuífero es libre y contiene agua de buena calidad. Este es el acuífero de donde se obtiene agua para abastecimiento público.

\section{Métodos}

\subsection{Método directo: muestreo del suelo contaminado}

El plan de muestreo se realizó de acuerdo con lo establecido en la NOM-138-SEMARNAT/SS-2003. Los puntos muestreados se definieron en campo de acuerdo con la inspección visual e información previa sobre el lugar, y tomando en cuenta el complejo nivel de acceso tratándose de un sitio industrial. Las muestras obtenidas fueron simples, de acuerdo con lo que señala la normatividad, además se obtuvo una muestra duplicada por cada 10 , sin utilizar lodos de perforación. Para tener un adecuado control en el muestreo, el personal encargado llevó una bitácora, hojas de campo para cada muestra obtenida y las cadenas de custodia, señalando los análisis químicos específicos para cada muestra.

Los puntos muestreados en el sitio contaminado fueron 22 en total (Figura 1), a distancias variables, entre la superficie y $3 \mathrm{~m}$ de profundidad en promedio, excepto el punto PUE18 que se muestreó a $5.20 \mathrm{~m}$ de profundidad debido a que se observó fuerte contaminación desde la superficie hasta ese punto. Estas profundidades se definen en campo por apreciación visual y organoléptica. En total se realizaron 42 muestras, incluidos 4 duplicados. Las muestras fueron recolectadas con la ayuda de un equipo neumático Long Year modelo 34, con motor diésel marca Perkings 


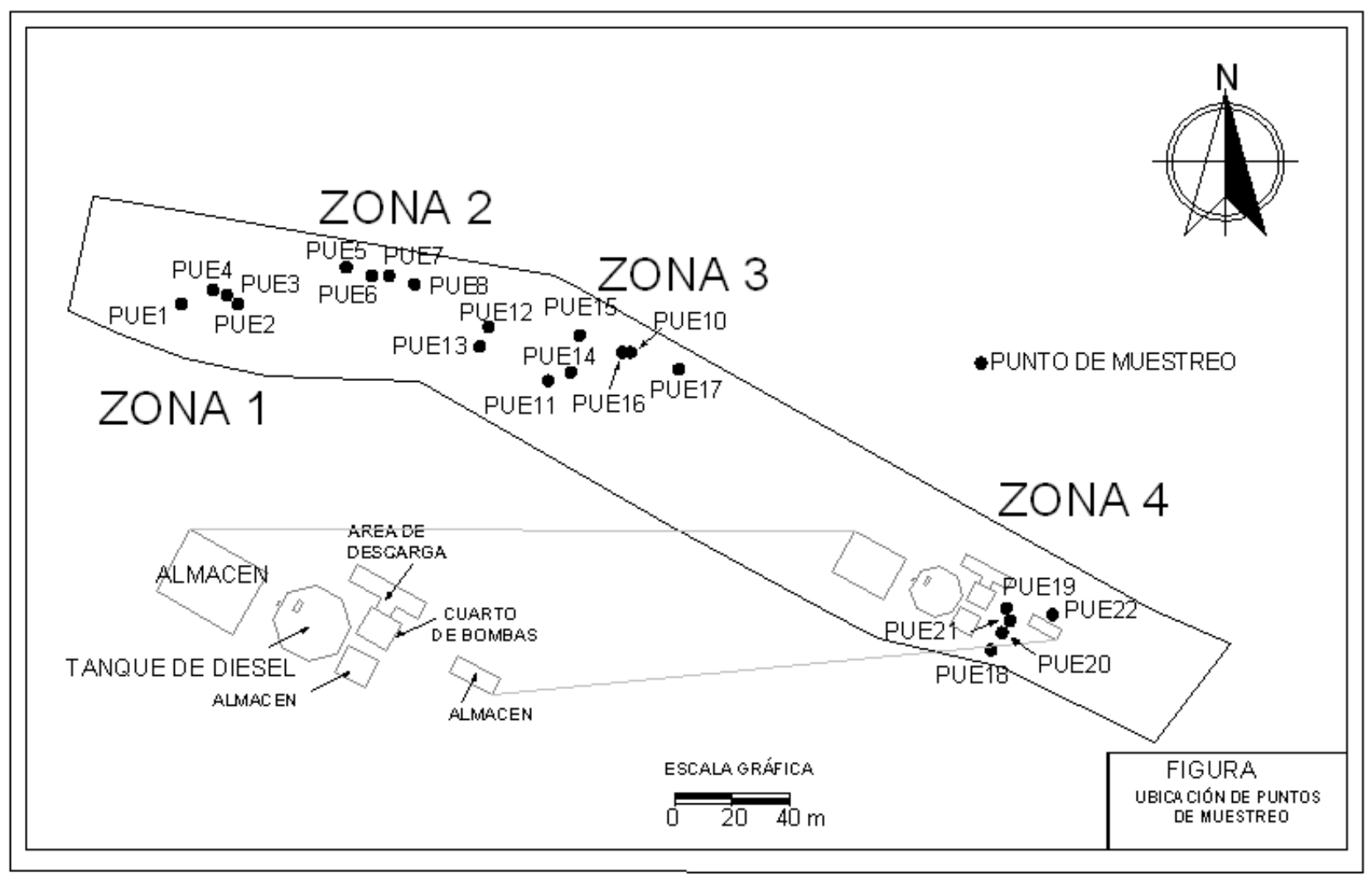

Figura 1. Ubicación de los puntos de perforación y toma de muestras de suelo.

que incluye un sistema para evitar la formación de chispa durante el encendido.

De acuerdo con la información adquirida a priori y a las observaciones realizadas, el diésel es el combustible más utilizado en las actividades desarrolladas en el sitio. La NOM-138-SEMARNAT/SS-2003 señala que cuando el sitio está contaminado con diésel deben analizarse los hidrocarburos fracción media (HFM) e hidrocarburos aromáticos polinucleares (HAP), por lo que se realizaron estos análisis con los métodos EPA8015B y EPA 8310, respectivamente.

Para los análisis tanto de HFM como para HAP se extrajo el suelo del tubo partido, se tamizó en malla del número $10 \mathrm{y}$ se colocó en un frasco de vidrio de $300 \mathrm{~mL}$ de capacidad. En los casos donde el suelo presentó alta humedad o saturación de hidrocarburos y/o cuando el suelo fue muy plástico, éste no fue tamizado y las muestras se obtuvieron del tubo de aluminio (liner). El tubo de aluminio se extrajo del tubo partido y se cortó en ambos extremos quedando de una longitud aproximada de $25 \mathrm{~cm}$. Posteriormente se colocó teflón y sobre éste cinta adhesiva. Más tarde se colocó una etiqueta en la superficie del tubo de aluminio o del frasco de vidrio con la identificación de la muestra, profundidad, fecha, hora, responsable del muestreo y análisis a realizar. Finalmente, los liners y los frascos de vidrio se colocaron dentro de una bolsa de plástico y ésta dentro de una hielera donde permanecieron a baja temperatura $\left(4{ }^{\circ} \mathrm{C}\right)$.
El volumen de suelo contaminado se calculó multiplicando el área del contorno por el espesor de la capa. Los análisis fueron realizados por los Laboratorios ABC. Química, Investigación y Análisis S.A. de C.V., con la aprobación de la Entidad Mexicana de Acreditación (EMA).

\subsection{Método Indirecto: aplicación del método ERT}

Para todos los perfiles, el equipo que se empleó para la adquisición de los datos geoeléctricos fue el SYSCAL Pro (Iris Instruments, 2012). Esta unidad de medición de resistividad está diseñada para toma de datos masiva. El equipo contiene un transmisor, receptor y alimentación, por lo que es una herramienta muy práctica en campo.

En la zona 3 del área de estudio se realizaron seis perfiles paralelos con orientación E-O, para obtener los datos de la tomografía eléctrica (Figura 2) con arreglo electródico dipolo-dipolo. En cada perfil se colocaron 41 electrodos, utilizando un cable inteligente de 24 electrodos, con un espaciamiento entre ellos de $0.5 \mathrm{~m}$ y una separación entre cada línea de $4 \mathrm{~m}$. Análogamente se realizaron dos líneas adicionales en los puntos con mayor contaminación (PUE1A y PUE18A), zona 1 y 4 respectivamente (Figura 2). Para obtener estos perfiles se utilizaron 34 electrodos con un espaciamiento entre ellos de $0.75 \mathrm{~m}$. El arreglo fue Wenner-Schlumberger. En el lugar se obtuvo muestra de suelo en dos sitios diferentes: uno contiguo al perfil seis y 


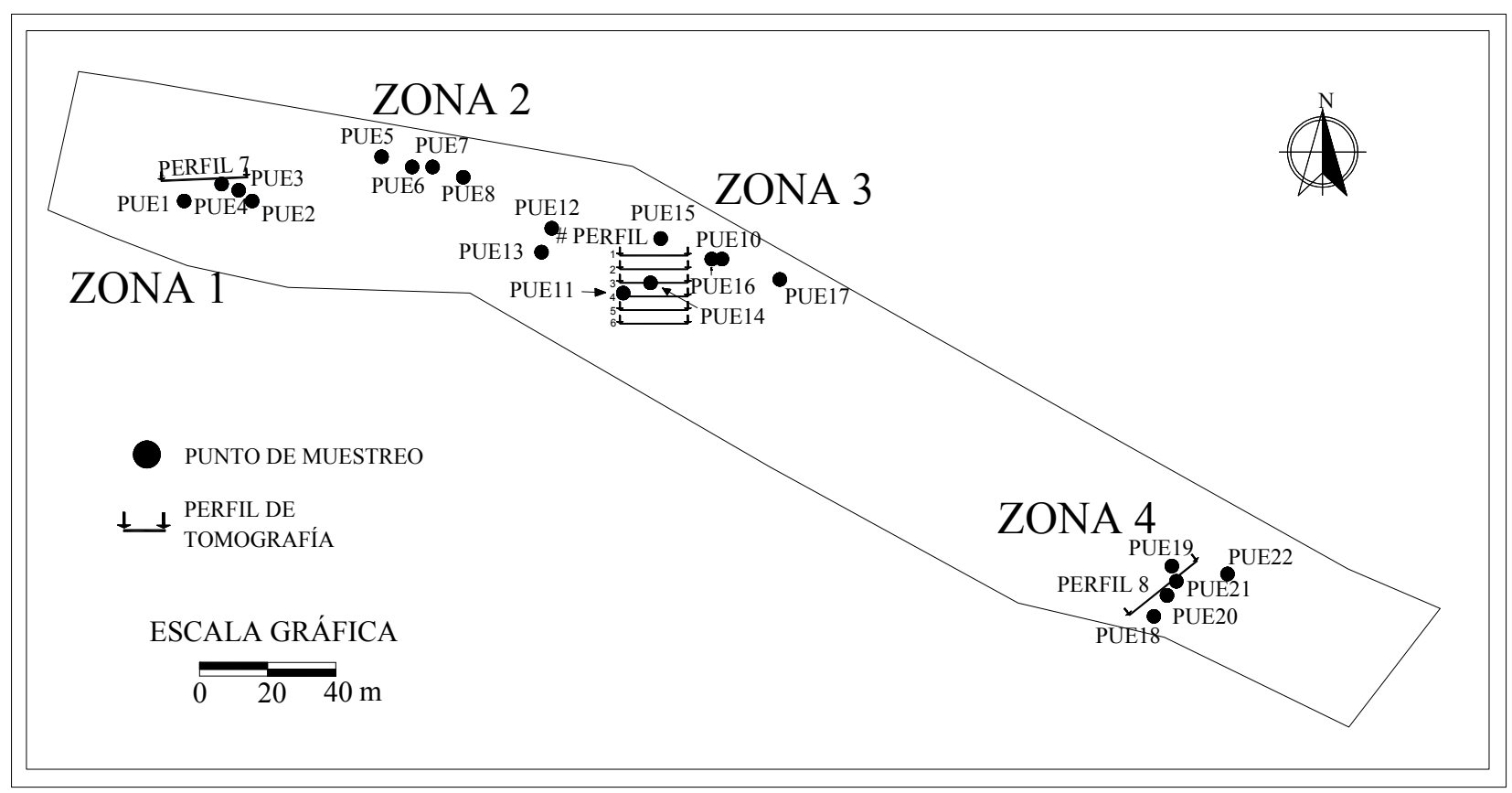

Figura 2. Ubicación de perfiles geofísicos.

el segundo a un metro del perfil PUE1, con la finalidad de realizar el análisis granulométrico.

Para la inversión de los datos se utilizó el software RES2DINV (Loke y Barker, 1996); los resultados obtenidos de los modelos de inversión se exportaron a SURFER para lograr una mejor visualización. En el caso de la inversión tridimensional de los datos se utilizó el software RES3DINV (Loke y Barker, 1996); posteriormente los datos se exportaron al VOXLER para generar el cubo de resistividad.

\section{Resultados y discusión}

4.1.Resultados de la caracterización del suelo contaminado por el método directo

\subsubsection{Hidrocarburos Fracción Media (HFM)}

Nueve muestras de suelo de un total de 42 (21\%) (Tabla 1), registran valores por arriba de los $5000 \mathrm{mg} / \mathrm{kg}$, valor que estipula la norma NOM-138-SEMARNAT/SS-2003 como límite máximo permisible para HFM. Los valores que exceden dicho límite, oscilan entre $5468 \mathrm{mg} / \mathrm{kg}$ y 36565 $\mathrm{mg} / \mathrm{kg}$. En la misma tabla se muestran todos los puntos que tuvieron concentración de hidrocarburos.

El área más afectada (a profundidad) se ubica en la zona 4, donde se encuentran los tanques elevados, aunque la muestra con mayor concentración es PUE1 (36565 mg/kg), que se encuentra entre la superficie y $2 \mathrm{~m}$ de profundidad.

\subsubsection{Hidrocarburos Aromáticos Polinucleares (HAP)}

Se realizó el análisis sólo en las zonas donde se apreció contaminación visual. Se observa presencia de estos en 12 de
Tabla 1. Puntos que rebasan las concentraciones de hidrocarburos fracción media (NOM-138-SEMARNAT/SS-2003).

\begin{tabular}{cccc}
\hline NÚM. & IDENTIF. & PROF (m) & HFM (mg/kg) \\
\hline 1 & PUE1 & 1.2 & $\mathbf{3 6 5 6 5}$ \\
9 & PUE6 & 0.9 & $\mathbf{2 4 6 7 8}$ \\
14 & PUE10 & 0.6 & 579 \\
15 & PUE11 & 0.6 & 4145 \\
16 & PUE12 & 0.6 & 727 \\
22 & PUE15 & 1.3 & $\mathbf{7 2 2 7}$ \\
23 & PUE15 & 1.8 & 462 \\
24 & PUE15 & 2.5 & 2964 \\
27 & PUE18 & 1.3 & $\mathbf{7 ~ 0 4 8}$ \\
28 & PUE18 & 2.2 & $\mathbf{5 4 6 8}$ \\
29 & PUE18 & 2.3 & 3991 \\
30 & PUE18 & 3.8 & $\mathbf{8 5 5 1}$ \\
31 & PUE18 & 4.9 & $\mathbf{9 3 1 7}$ \\
32 & PUE18 & 5.2 & $\mathbf{6 ~ 1 0 8}$ \\
35 & PUE20 & 1.6 & $\mathbf{9 9 0 3}$ \\
37 & PUE20 & 3.8 & 1814 \\
39 & PUE22 & 1.4 & 213 \\
\hline
\end{tabular}

las 20 muestras analizadas; sin embargo, todas se encuentran dentro de los límites máximos permisibles de la NOM-138SEMARNAT/SS-2003 (el valor es de $10 \mathrm{mg} / \mathrm{kg}$ base seca para uso industrial de todos los hidrocarburos aromáticos polinucleares que contempla la norma, con excepción del benzo(k)fluoranteno que es de $80 \mathrm{mg} / \mathrm{kg}$ ).

\subsubsection{Cálculo de áreas y volúmenes de suelo contaminado}

Para el modelado espacial de la zona de estudio se utilizó el software SURFER. La salida del modelo es para 
una sola profundidad, por lo que se consideraron tres capas: 1) entre la superficie y $3 \mathrm{~m}$ de profundidad; 2) entre $3 \mathrm{~m}$ y $5 \mathrm{~m}$ de profundidad; y 3 ) más de $5 \mathrm{~m}$ de profundidad. Los volúmenes que requieren remediación se calcularon a partir de las áreas obtenidas con el modelo en SURFER y con las profundidades a las que se obtuvieron las muestras con presencia de HFM (Tabla 2). En las Figura 3a, b 3b y $3 \mathrm{c}$ se encuentran las zonas configuradas y los volúmenes
Tabla 2. Áreas y volúmenes contaminados con hidrocarburos fracción media entre la superficie y $>5 \mathrm{~m}$ de profundidad.

\begin{tabular}{|c|c|c|}
\hline $\begin{array}{l}\text { Intervalo de profundidad } \\
\text { (m) }\end{array}$ & $\begin{array}{r}\text { Área } \\
\left(\mathbf{m}^{2}\right)\end{array}$ & $\begin{array}{c}\text { Volumen } \\
\left(\mathbf{m}^{3}\right)\end{array}$ \\
\hline $0-3$ & 341 & 1026 \\
\hline 03-may & 116 & 232 \\
\hline$>5$ & 43 & 43 \\
\hline TOTAL & 500 & 1301 \\
\hline
\end{tabular}

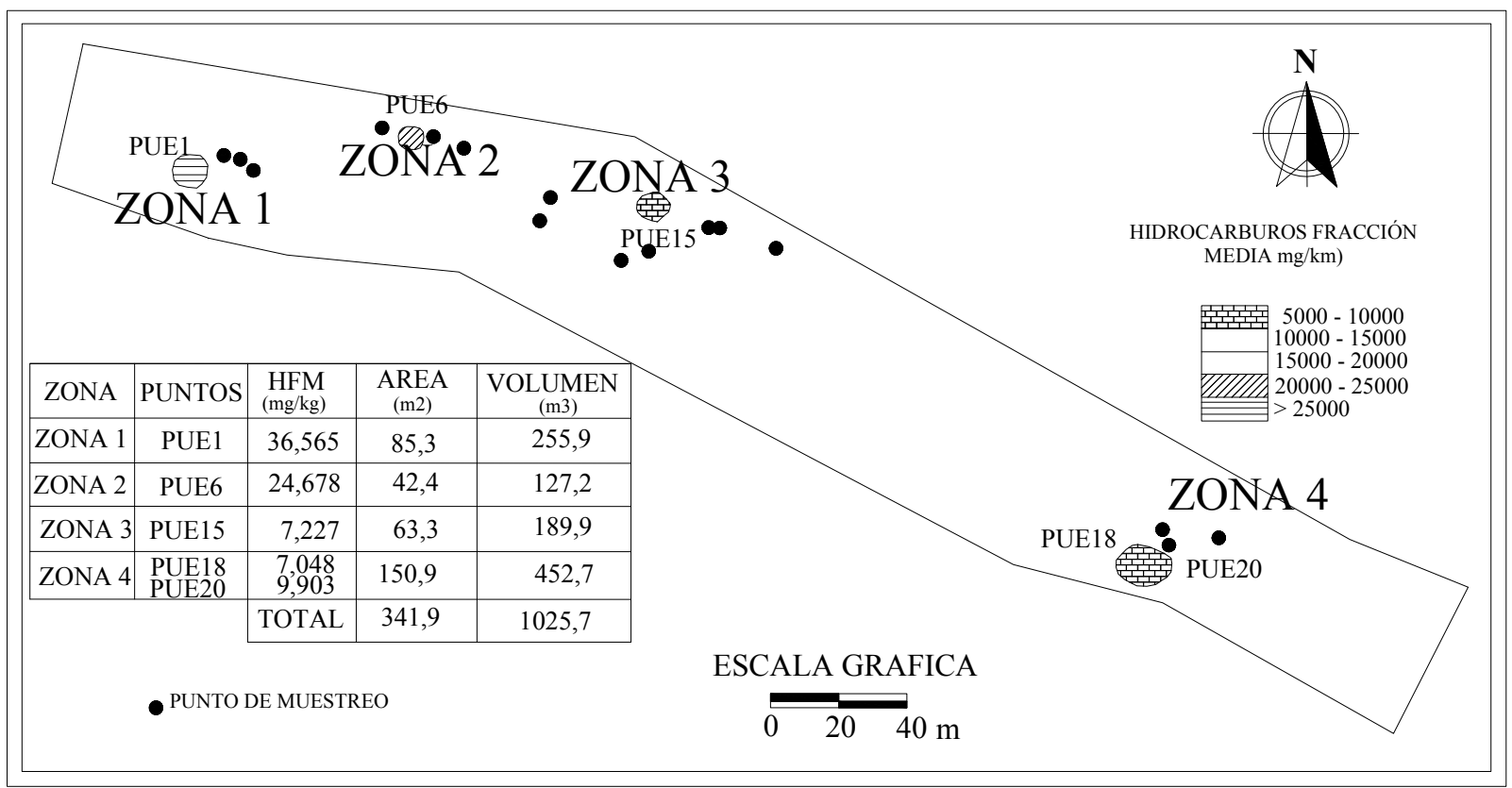

a)

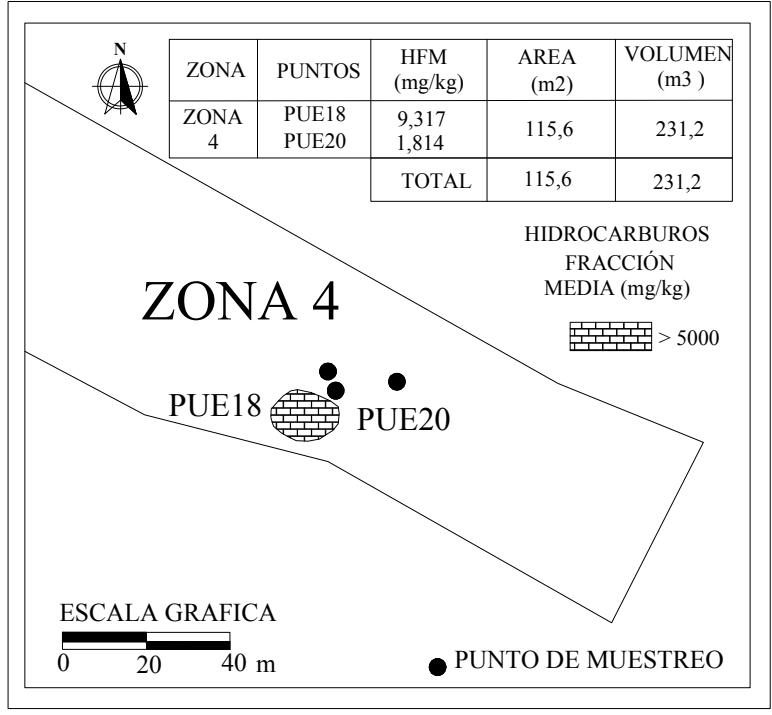

b)

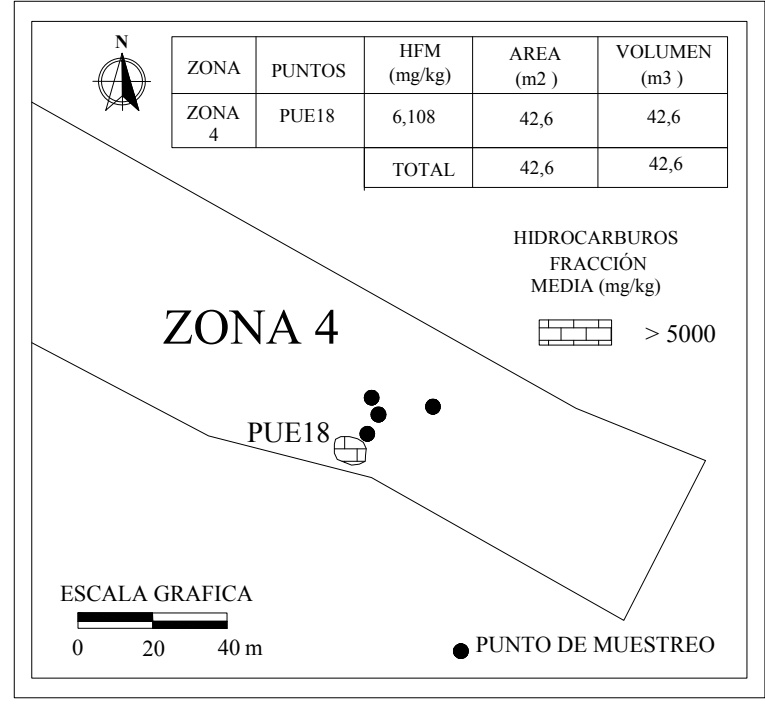

c)

Figura 3. a) Configuración de contaminación por HFM a profundidad de 0 - $3 \mathrm{~m}$; b) Configuración de contaminación de HFM a profundidad de 3 - 5 m; y c) Configuración de contaminación de HFM a profundidad mayor de $5 \mathrm{~m}$. 
calculados.

El volumen total de suelo que sobrepasa la NOM-138SEMARNAT/SS-2003 para HFM entre la superficie y $6 \mathrm{~m}$ de profundidad es de $1301 \mathrm{~m}^{3}$.

\subsection{Resultados de la caracterización del suelo} contaminado por el método indirecto

\subsubsection{Inversión de los perfiles de resistividad}

A continuación se interpretan los perfiles realizados en el sitio de acuerdo con sus propiedades de resistividad y la delimitación de los contaminantes. En la Figura 4 se muestran los perfiles del 1 al 6 . En los perfiles 1 a 4 se puede observar, que a partir del primer metro de profundidad se encuentran valores altos de resistividad (entre 600 y $1000 \mathrm{Ohm}-\mathrm{m}$ ) como evidencia de la alta resistividad del material de relleno compactado en el sitio; aunque la sección geoeléctrica del perfil 4 no incluye al pozo PUE11, su proximidad y la contaminación detectada en el mismo $(4145 \mathrm{mg} / \mathrm{kg}$ a $0.6 \mathrm{~m}$ de profundidad) indica la presencia de contaminación reciente en el subsuelo. El terreno natural (las zonas en color verde) del área tiene resistividades entre 25 y 40 Ohm-m.

A diferencia de los perfiles anteriores, en el perfil 5 desaparece por completo el alto resistivo en la superficie; sin embargo aparece una concentración a partir de aproximadamente $1 \mathrm{~m}$ hasta llegar a $1.5 \mathrm{~m}$. No es tan marcado como las anteriores y se logra ver con mayor claridad el terreno natural.

$\mathrm{Al}$ igual que en el perfil 5, en el 6 el alto resistivo se observa únicamente del lado izquierdo del perfil y no se presenta desde la superficie, empieza a partir de $0.5 \mathrm{~m} \mathrm{y}$ hasta $2.5 \mathrm{~m}$ de profundidad. Este valor de resistividad va disminuyendo hacia el lado derecho del perfil indicando la resistividad del medio natural, al igual que los perfiles anteriores en color verde.

El perfil 7 se encuentra ubicado cerca del punto de muestreo PUE1 (Figura 5), en el sitio directamente se logró observar el hidrocarburo (diésel) aflorando y el perfil quedó paralelo a esta mancha; nuevamente las altas resistividades superficiales pueden incluir el efecto de la contaminación reciente. Se presenta una franja conductiva, a partir de $1.5 \mathrm{~m}$ de profundidad y de los $8 \mathrm{~m}$ de distancia horizontal, siendo probablemente presencia de contaminación madura, es decir, afectada por los procesos de biodegradación, tomando en cuenta las características litológicas del sitio (suelo arenoso). En la parte superior del perfil, se delinean zonas con mayor resistividad, disminuyendo con la profundidad hasta aproximadamente $1 \mathrm{~m}$.

El perfil 8 (Figura 6), se encuentra localizado entre dos tanques de almacenamiento de diésel. Se observa que cruza los puntos PUE18, PUE20, PUE21 y PUE 19. En el informe de los análisis de contenido de hidrocarburos para estos puntos, el más contaminado es el PUE18 con una concentración de $7048 \mathrm{mg} / \mathrm{kg}$ a una profundidad de $1.3 \mathrm{~m}$. Desafortunadamente, en la inversión se pierden esos datos, aunque se puede asumir que las zonas en color azul muestran la presencia de contaminación madura, debido a que en esa zona se mantienen activos los tanques de almacenamiento y existe la posibilidad de encontrar aporte de contaminantes al suelo por tiempo prolongado. Los demás puntos no rebasan el límite máximo permisible de la norma, sin descartar que exista contaminación.

Además de las configuraciones realizadas en SURFER, se utilizó el software Voxler (versión 2.0), para una mejor apreciación de la migración y la delineación de la contaminación, tanto lateral como a profundidad. Los datos utilizados fueron de los perfiles 1 a 6 a tres profundidades diferentes: $0.5 \mathrm{~m}, 1.0 \mathrm{~m}$ y $1.5 \mathrm{~m}$. En la Figura $7 \mathrm{se}$ graficaron los puntos de resistividad de los seis perfiles dispuestos. Se identifican las diferentes profundidades que se tomaron de los datos invertidos, para lograr su apreciación en 3D y poder visualizar la tendencia que presenta la contaminación. Los puntos rojos representan resistividad alta en el sitio, incluyendo la presencia de contaminación reciente. Las zonas en color verde y amarillo es el medio natural del sitio sin contaminación, mientras que las zonas en color azul representan la probable presencia de contaminación madura.

En la Figura 8 se logra observar los perfiles dispuestos en un cubo, de tal manera que la contaminación reciente es delineada en los ejes $x, y, z$. Los cruces en la configuración, ayudan a ver la extensión de la contaminación en tales zonas donde se presentan resistividades altas. El punto PUE14, el cual reportó concentración de contaminación, cae en la zona donde los datos del método indirecto indican presencia de contaminante.

En la Figura 9 se representa la configuración de la migración elaborada con los datos de tomografía eléctrica. En el área que envuelven los perfiles 1 al 4, se aprecia una mayor contaminación que hacia los perfiles 5 y 6 , donde la contaminación es más puntual. El punto PUE14, sigue cayendo dentro de la delineación de los altos resistivos. Para una mejor apreciación del comportamiento del contaminante, en la Figura 9 se muestra el medio encajonante del sitio, que son las zonas en color verde y amarillo que se encuentran combinadas con una anomalía de altas resistividades de color rojo, que se identifica como el hidrocarburo reciente presente en la zona. Se observa que hacia los perfiles 1 al 4, la mancha contaminante es mayor que hacia los perfiles 5 y 6 . En el perfil 6 (del lado este), se ve una acumulación puntual del contamínate. En el punto PUE11, al parecer el medio encajonó al contaminante en un punto aislado del derrame de arriba.

La Figura 10 es un plano $x-y$ a una profundidad de $0.5 \mathrm{~m}$, donde se logra apreciar una posible delineación de la pluma contaminante entre el perfil 2 y el perfil 3. Hacia el perfil 4, la correspondencia del punto PUE11 con un valor de 4145 $\mathrm{mg} / \mathrm{kg}$ de concentración de hidrocarburo muestreado a 0.6 $\mathrm{m}$, cae sobre una zona delineada con valores de hasta más de $1000 \mathrm{Ohm}-\mathrm{m}$ (zona roja), representando dicha presencia del contaminante. Las zonas que presentan resistividades entre 25 y $40 \mathrm{Ohm}-\mathrm{m}$, son aquellas que se consideran como 


\section{PERFIL 1}

PUE15

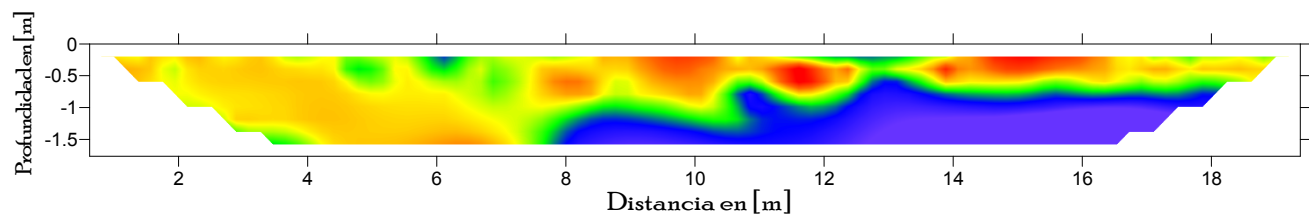

\section{PERFIL 2}

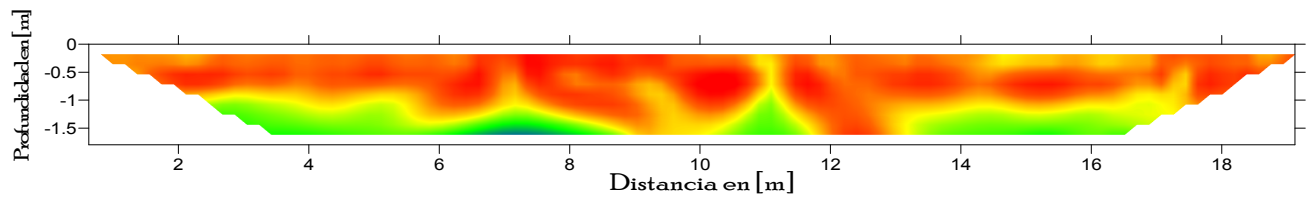

\section{PERFIL3}

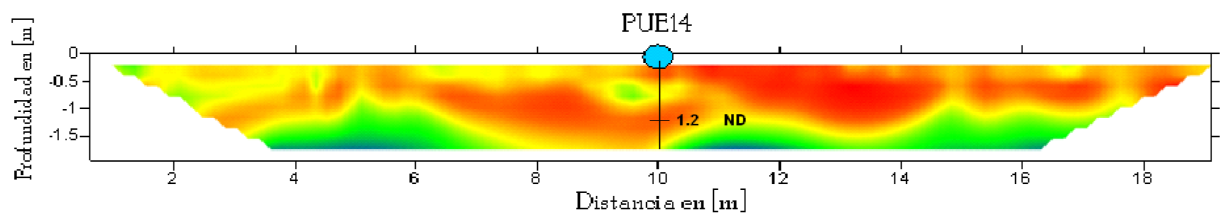

PERFIL 4

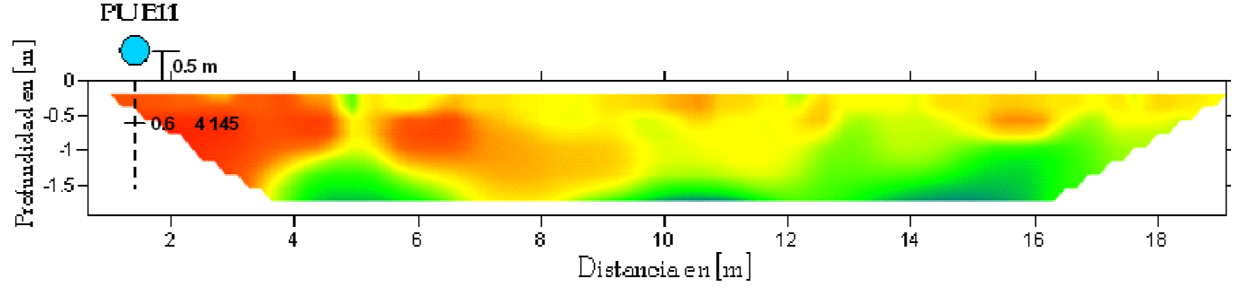

PERFIL 5

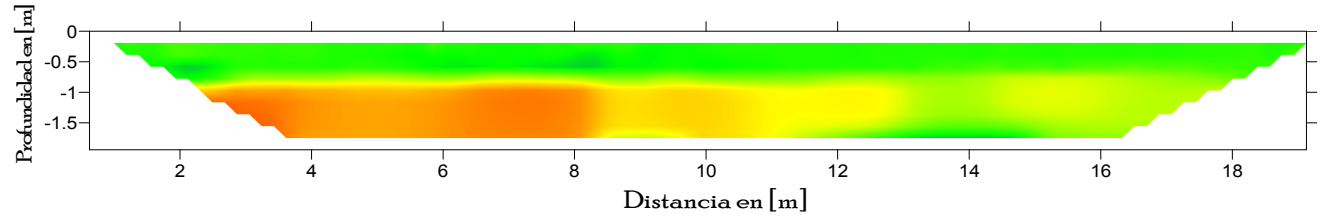

\section{PERFIL 6}
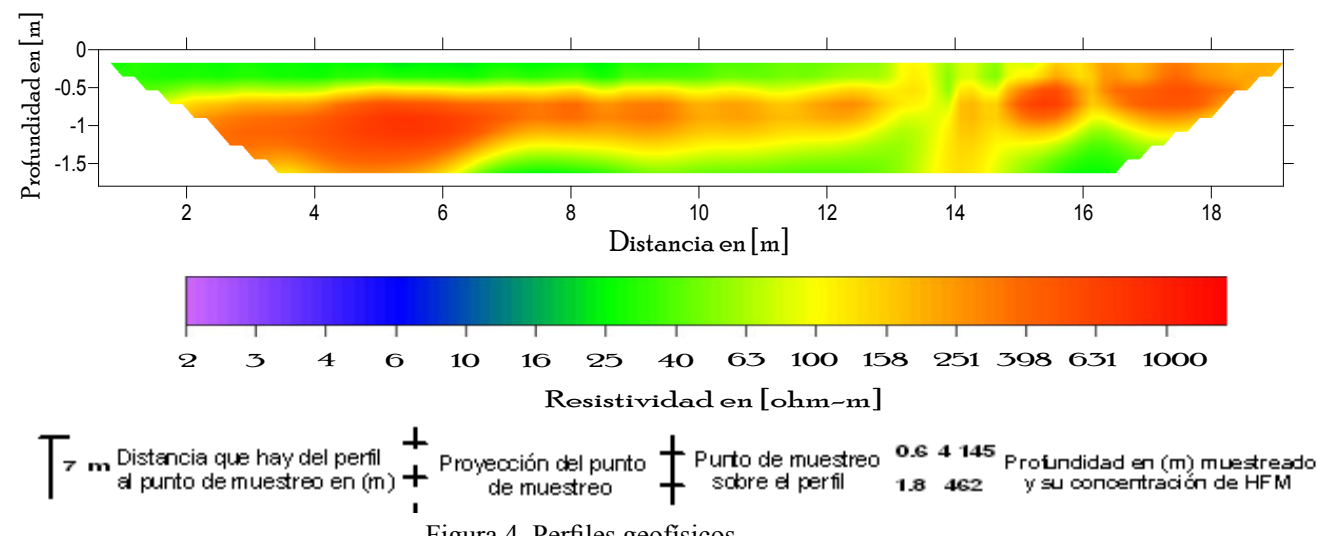

Figura 4. Perfiles geofísicos. 


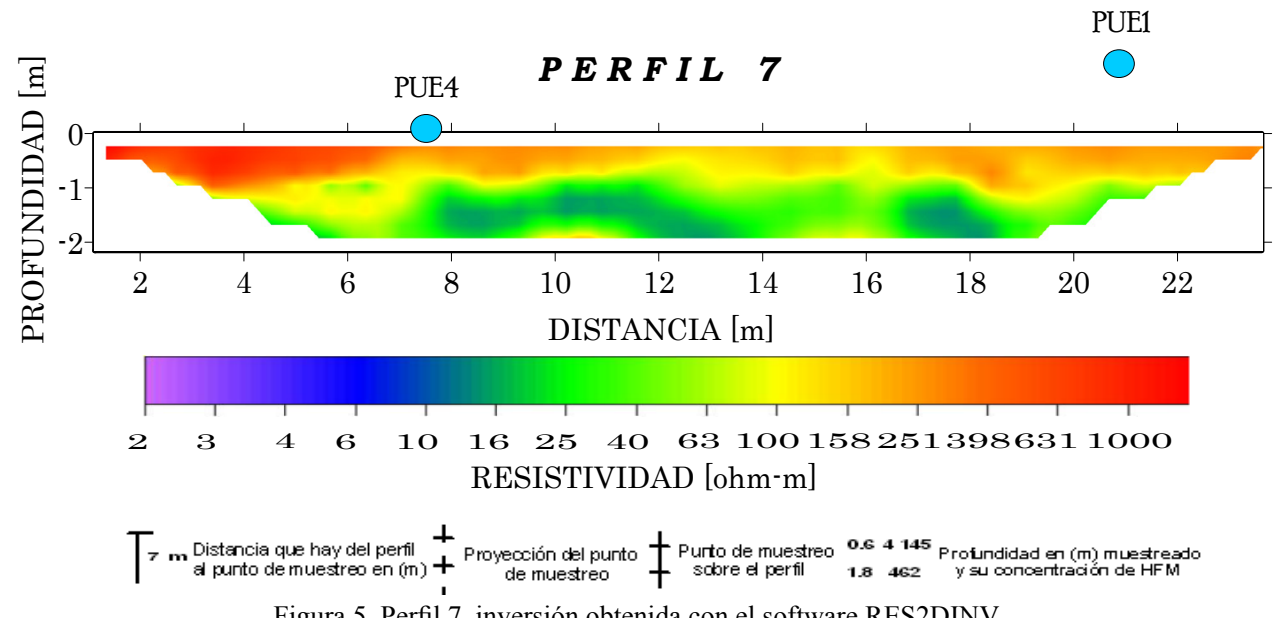

Figura 5. Perfil 7, inversión obtenida con el software RES2DINV.

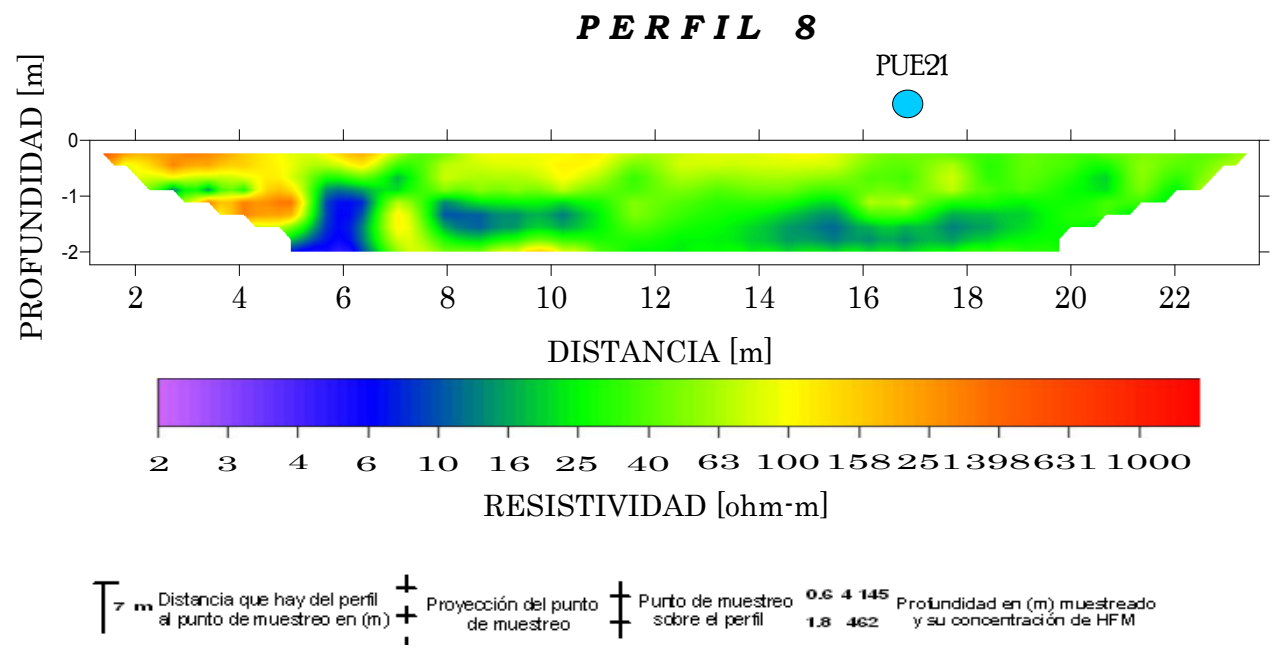

Figura 6. Perfil 8, inversión obtenida con el software RES2DINV.

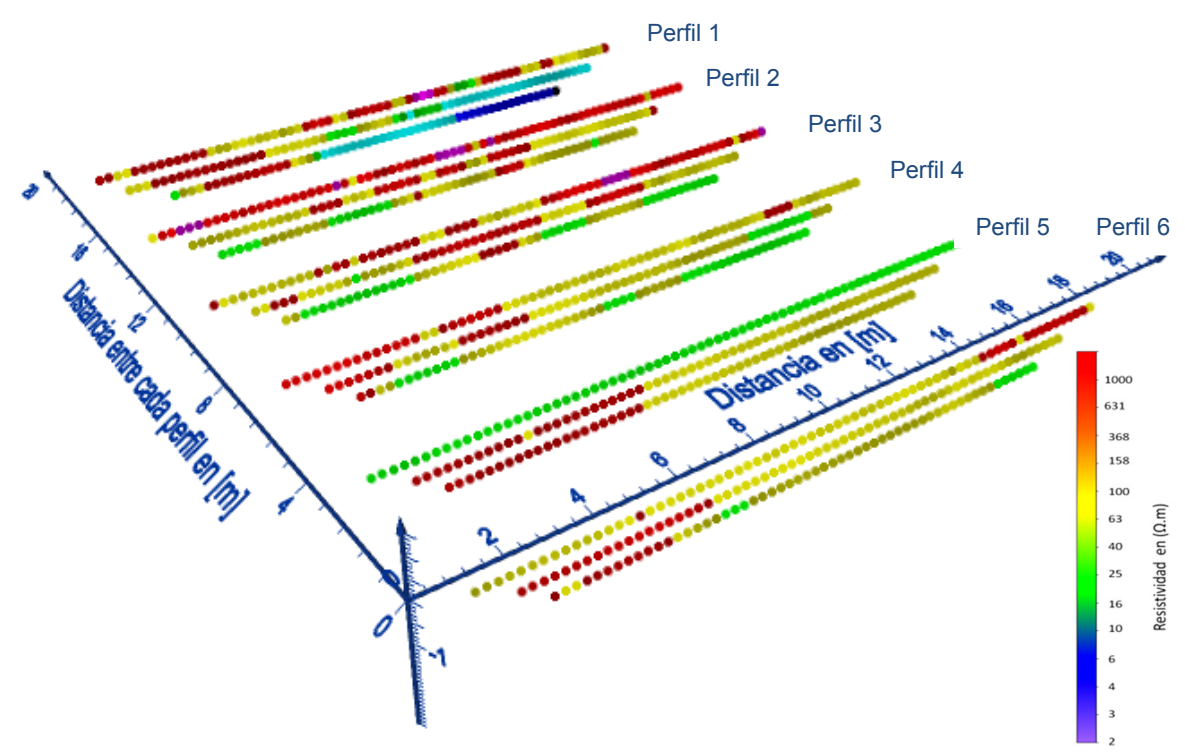

Figura 7. Puntos de los datos de resistividad en Ohm-m, de los 6 perfiles. 


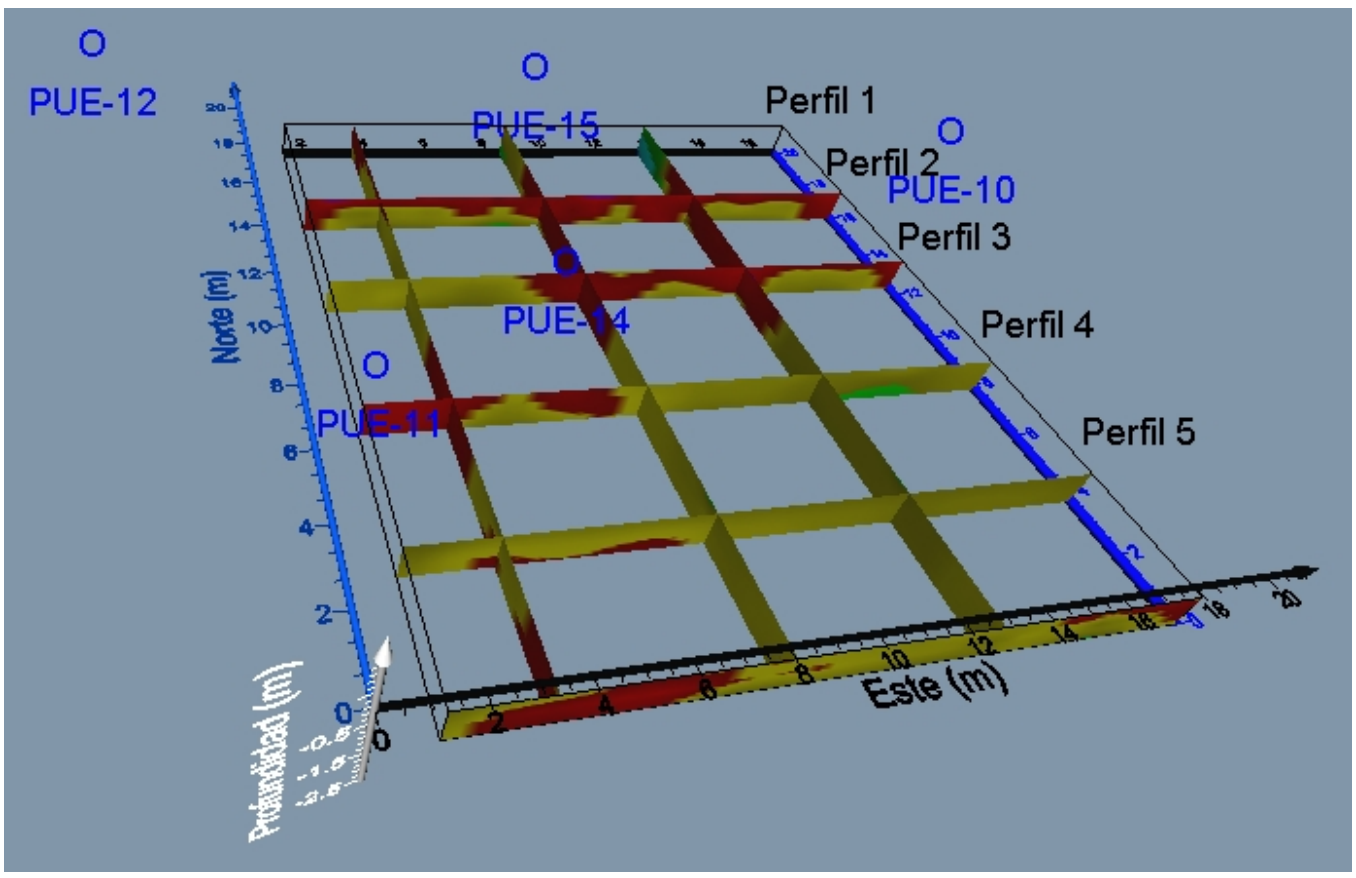

Figura 8. Delineación de la contaminación en la zona 3.

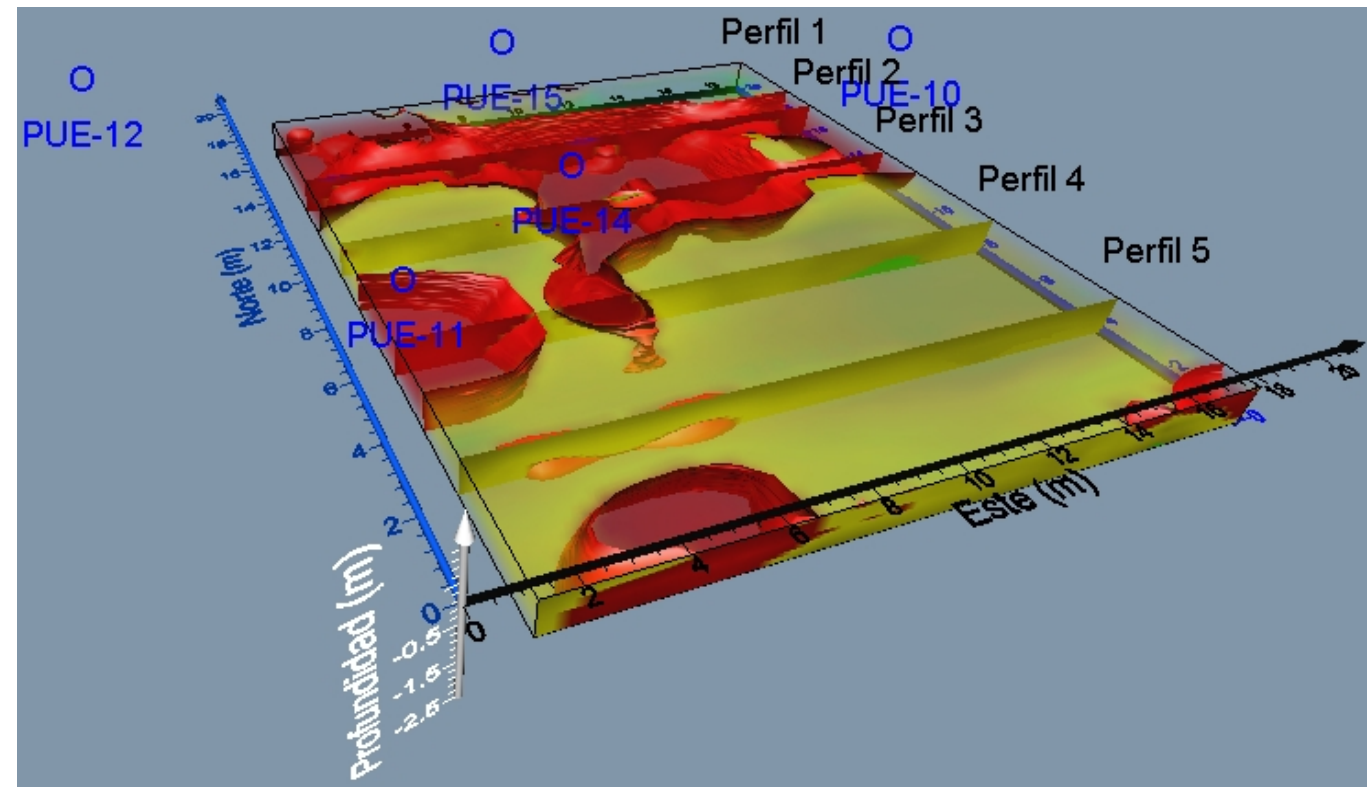

Figura 9. Delineación de la contaminación y del medio encajonante.

el medio natural sin contaminación, presentes en la parte centro-este, y el perfil 5 delinea una zona de entre 16 y 40 Ohm-m. También se logran apreciar zonas más puntuales de la contaminación en la zona estudiada.

La Figura 11 es un plano $x-y$ a una profundidad de 1.0 $\mathrm{m}$, se puede interpretar una disminución en la mancha del contaminante entre los perfiles 2 y 3 , aunque la concentración se manifiesta en el centro. Se observa la muestra PUE14 que se ubica en una zona de contaminación y sin embargo no reporta concentración a esta profundidad de $1.1 \mathrm{~m}$. Se puede justificar que la muestra fue tomada justo en una pequeña interfaz donde la contaminación no fuera significativa y el laboratorio no reportara un dato de concentración. Los puntos aislados siguen presentándose en la imagen, además de la zona del centro-este que parece mantenerse sin contaminación.

En la Figura 12, a una profundidad de $1.5 \mathrm{~m}$, en el plano $x-y$ se identifica un área más pequeña de la pluma contaminante entre los perfiles 2 y 3 , en comparación con la Figura 11. Para el caso del perfil 4, la contaminación sigue 


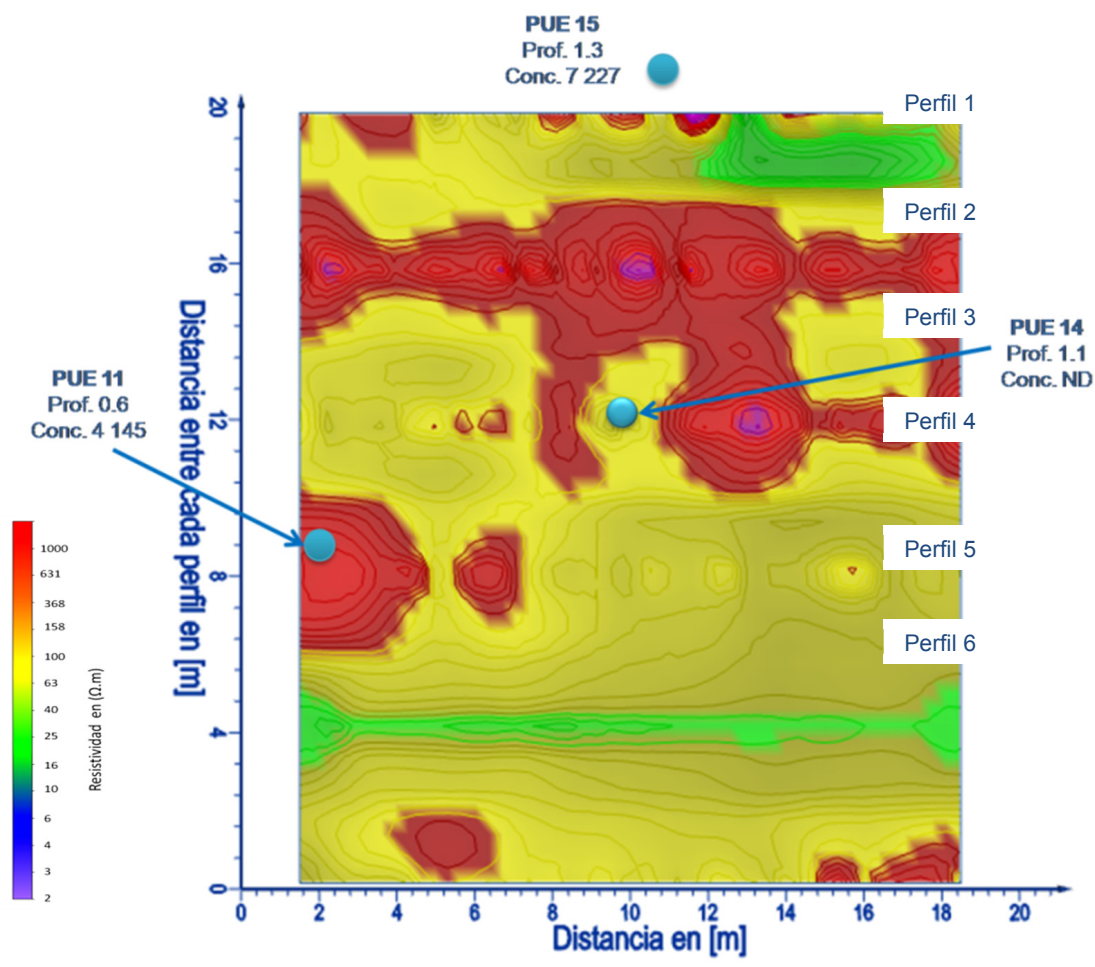

Figura 10. Delineación de la contaminación en un plano $x-y$ a $0.5 \mathrm{~m}$ de profundidad (zonas rojas).

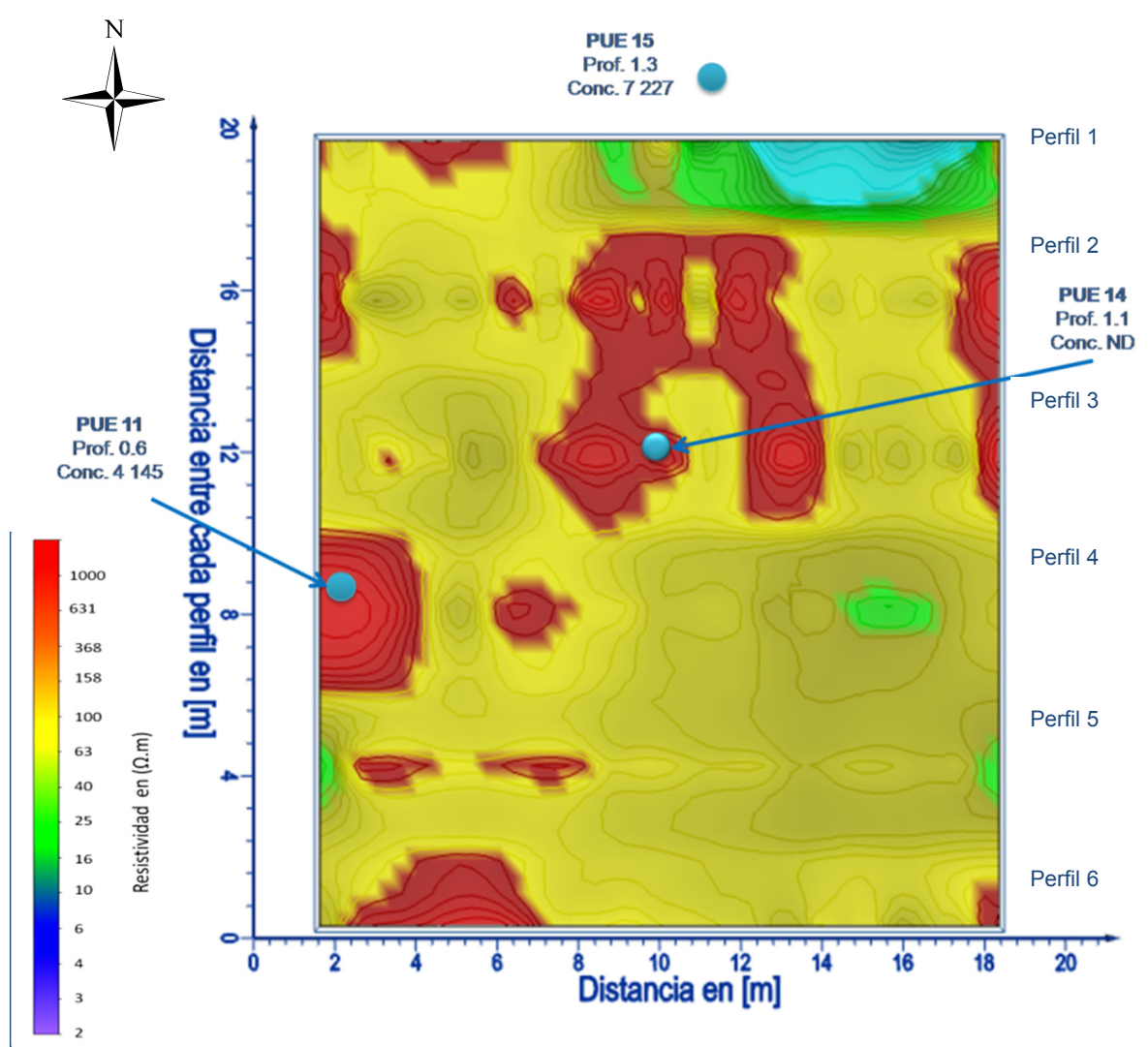

Figura 11. Delineación de la contaminación en un plano $x-y$ a $1.0 \mathrm{~m}$ de profundidad (zonas rojas). 


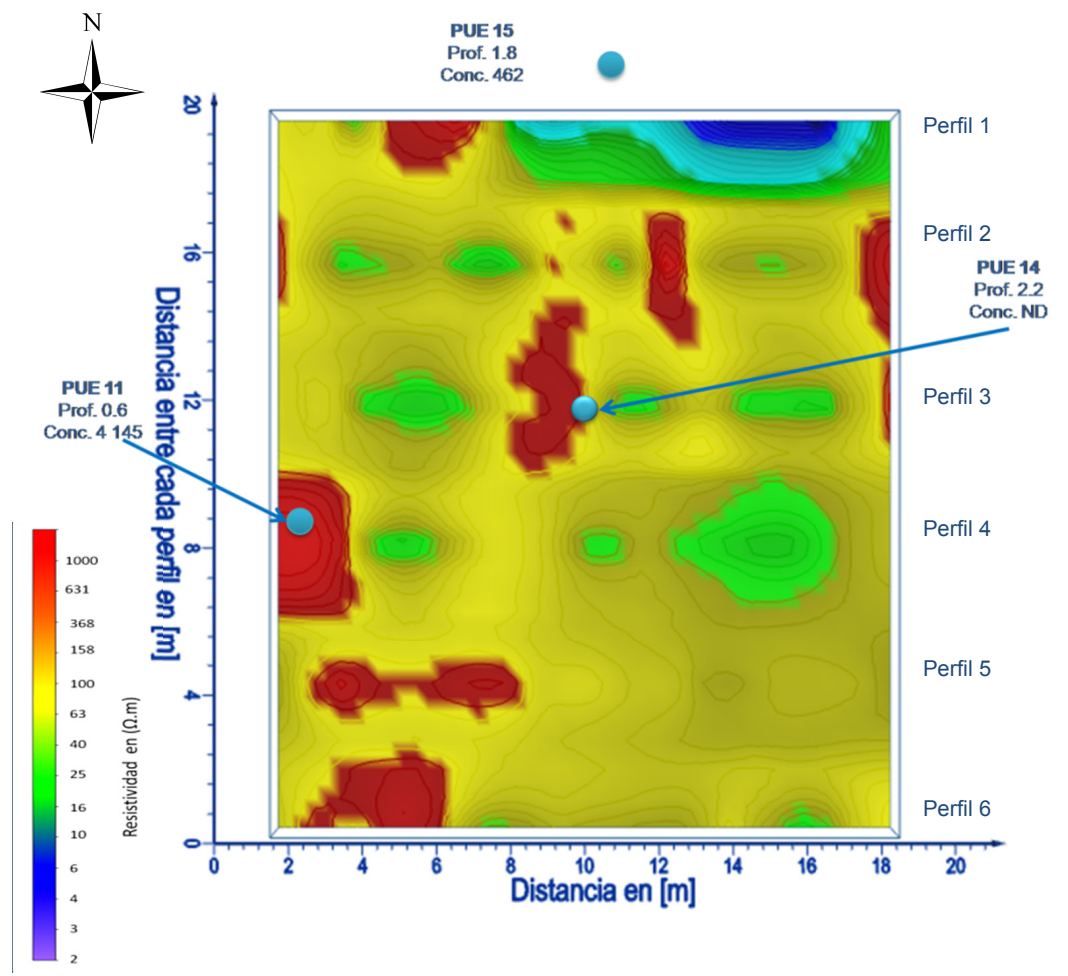

Figura 12. Delineación de la contaminación en un plano $x-y$ a $1.5 \mathrm{~m}$ de profundidad (zonas rojas).

apareciendo y en el perfil 5 se incrementa la delineación de la zona roja en su lado oeste al igual que en el perfil 6, definiendo la misma forma que en la Figura 11. La zona limpia se muestra en la mayor parte de la zona estudiada, con puntos aislados de pequeñas manchas con resistividades altas.

Estas configuraciones ayudan a observar las zonas que presentan un intervalo de entre $300 \mathrm{Ohm}-\mathrm{m}$ y $1000 \mathrm{Ohm}-\mathrm{m}$, que son zonas que tienen un menor grado de contaminación y/o impregnación del hidrocarburo, a diferencia de zonas con valores arriba de los $1000 \mathrm{Ohm}-\mathrm{m}$ que pudieran deberse a concentraciones mayores.

\subsubsection{Correlación del método directo (muestreo) y el método indirecto (tomografia eléctrica)}

Tomando en cuenta los pocos puntos que se logran correlacionar con los perfiles de tomografía eléctrica, se identifica que el alto resistivo (valores entre 600 y 1000 Ohm-m) son zonas con una mayor problemática de contaminación, que va disminuyendo a profundidad conforme la resistividad decrece.

En la zona 1 (perfil 7), cuya esquematización fue aproximada y puntual de acuerdo con las observaciones de campo y con los resultados reportados, el punto PUE1 reporta una contaminación de $36,565 \mathrm{mg} / \mathrm{kg}$ a una profundidad de $1.2 \mathrm{~m}$.

En la zona 3 se esquematizan las concentraciones a dos profundidades diferentes: una de 0 a $1 \mathrm{~m}$ de profundidad y de 1 a $2 \mathrm{~m}$. En la configuración de 0 a $1 \mathrm{~m}$ se tomaron los puntos PUE10, PUE11 y PUE12; el que presenta mayor concentración es el punto PUE11 con 4,145 mg/kg, logrando correlacionar el punto con la contaminación que reporta valores de alta resistividad (contaminación reciente).

Para las concentraciones encontradas entre 1 y $2 \mathrm{~m}$ fue en los puntos PUE13, PUE14, PUE15, PUE16 y PUE17. La mayor concentración en esta zona la tiene el punto PUE15 con $7227 \mathrm{mg} / \mathrm{kg}$. Las dos configuraciones abarcan parte de los perfiles paralelos (Perfil1 a perfil6).

En el caso de la zona 4 también se configuraron las concentraciones a dos intervalos de profundidades: una de 0 a $1.5 \mathrm{~m}$ y la otra de $1.5 \mathrm{~m}$ en adelante. En el primero se tomaron en cuenta los puntos PUE18, PUE20 y PUE22, donde el punto más contaminado es el PUE20 con una concentración de $9903 \mathrm{mg} / \mathrm{kg}$ a una profundidad de $1.6 \mathrm{~m}$. Para la profundidad de $1.5 \mathrm{~m}$ en adelante, los puntos configurados fueron PUE18 y PUE20, el punto más contaminado es el primero con $7048 \mathrm{mg} / \mathrm{kg}$ a una profundidad de $1.3 \mathrm{~m}$. El punto 18 se muestreó hasta una profundad de $5.2 \mathrm{~m}$, encontrando una concentración de $6108 \mathrm{mg} / \mathrm{kg}$, aunque el perfil sólo llega a una profundidad de investigación de aproximadamente $2 \mathrm{~m}$. El perfil que se correlaciona con esta zona es el perfil 8, que pasa sobre el punto reportado más contaminado (PUE18), donde se delinea una franja más conductiva (contaminación madura).

En los perfiles realizados son pocos los puntos contaminados con los que se puede hacer una comparación 
cuantitativa. En los perfiles del 1 al 6, correspondiente a la zona 3, solamente se tiene a los puntos PUE15 y PUE 11 con concentración de hidrocarburos. En el caso del perfil 7 en la zona 1, únicamente el punto PUE1 tiene concentración de hidrocarburos; y por último, en el perfil 8 de la zona 4, se cuenta con los puntos PUE18 y PUE20.

Las características del medio donde se realizaron los perfiles del 1 al 6, de la zona 3, es un suelo de relleno que fue depositado para la nivelación de las instalaciones y de acuerdo a su análisis, es un suelo arenoso con contenido importante de gravas y cantos rodados.

En general, se puede decir que a partir del primer metro de profundidad se encuentran valores altos de resistividad (entre 600 y $1000 \mathrm{Ohm}-\mathrm{m}$ ). Esta anomalía se infiere de la observación de la predominancia de un material con un valor de entre 25 y $60 \mathrm{Ohm}-\mathrm{m}$, definido como el material del terreno natural, con valores característicos de las arenas (Orellana, 1982), ya que se encuentra regularmente hacia la base de los perfiles y éste parece impregnado de un fluido externo a este (tal como el hidrocarburo).

El único punto de muestreo que se observó con concentración de hidrocarburos que pudiera corresponder a un alto resistivo en la zona 3 , es el punto PUE11 con una concentración de $4145 \mathrm{mg} / \mathrm{kg}$; pero la misma resistividad en el perfil 3 se observó en el punto PUE14 que indicó una concentración no detectada, aunque como se mencionó arriba pudiera haber caído la muestra en una zona con insignificante presencia de contaminación no detectada en el análisis de dicha muestra.

El bajo resistivo se muestra con el color azul. Los bajos resistivos generalmente en otras áreas se asocian con lentes arcillosos; sin embargo, en la zona de estudio el porcentaje de arcilla es aproximadamente del $1 \%$, por lo que la baja resistividad no es debido a esta litología. De esta manera se puede inferir la presencia de contaminación madura, es decir, afectada por los procesos de biodegradación, aunque esto requiere ser confirmado a partir de nuevas perforaciones y extracción de muestras de suelo para su análisis en laboratorio.

\section{Conclusiones}

Con el método de muestreo directo se obtiene que de las 42 muestras de suelo analizadas, nueve sobrepasan los límites máximos permisibles de HFM establecidos en la NOM-138-SEMARNAT/SS-2003; para el caso de los HAP, ninguna muestra excede los valores establecidos.

De acuerdo con las configuraciones realizadas con la ayuda del muestreo, se observa que la contaminación es puntual y el volumen de suelo contaminado calculado fue de $1301 \mathrm{~m}^{3}$. No se aprecia una migración del contaminante a mayores profundidades ni extensión en el área, a excepción del punto PUE18, en el que se detectó contaminación hasta una profundidad de $6.0 \mathrm{~m}$.

La contaminación reciente por hidrocarburos se encuentra en la parte más somera del corte, donde el diésel es vertido directamente en la superficie del terreno, donde un relleno altamente resistivo (entre 500 y $1000 \mathrm{Ohm}-\mathrm{m}$ ) se hace presente en la mayoría de los perfiles de ERT. Las zonas limpias del sedimento arenoso natural se identifican con valores de entre 25 y $150 \mathrm{Ohm}-\mathrm{m}$, en dependencia de la humedad del mismo. El método ERT confirma que al menos la contaminación reciente es puntual; sin embargo, en la configuración con el software Voxler se logra delinear un flujo y/o tendencia de la migración del contaminante, tanto lateralmente como a profundidad. Se delinean las zonas que pudieran tener una mayor concentración del contaminante y las zonas más limpias; logrando así, demostrar que la técnica es de gran utilidad para proponer puntos de muestreo directo que identificaran la presencia del contaminante. En 2D se logra visualizar el corte de cómo se comporta el contaminante, aunque la visualización en 3D da un mejor análisis de cómo y hasta donde podía extenderse dicha contaminación.

Los perfiles de ERT 7 y 8 muestran una capa geoeléctrica de baja resistividad $(\rho<10 \mathrm{Ohm}-\mathrm{m})$, lo que, de acuerdo a la información litológica del sitio, no se debe a un aumento del contenido de arcilla, siendo la causa más probable es la existencia de contaminación madura, afectada por los procesos de biodegradación, aunque se requiere de nuevas perforaciones y análisis químicos en muestras de suelo para su confirmación.

\section{Agradecimientos}

Los autores agradecen a la Dra. Claudia Arango Galván y a la M. en C. Guillermina Pérez Casimiro, su ayuda en los trabajos de Geofísica.

\section{Referencias}

Abdel Aal, G.Z., Atekwana, E.A., Slater, L.D., Atekwana, E.A., 2004, Effects of microbial processes on electrolytic and interfacial electrical properties of unconsolidated sediments: Geophysical Research Letters, 31, L12505, DOI:10.1029/2004gl020030.

Atekwana, E.A., Sauck, W.A., Werkema, Jr., D.D., 2000, Investigations of geoelectrical signatures at a hydrocarbon contaminated site: Journal of Applied Geophysics, 44, 167-180.

CONAGUA, 2009, Actualización de la disponibilidad media anual de agua subterránea, Acuífero (2104) Valle de Puebla: Subdirección General Técnica, Gerencia de Aguas Subterráneas, Subgerencia de Evaluación y Ordenamiento de Acuíferos, publicado en 28 de agosto del 2009, disponible en <www.conagua.gob. $\mathrm{mx}>$, consultado en febrero de 2013.

Dehaini, J., Aquino, W.F., Benetti-Mendes, J.M., 2000, The signal of GPR at a hydrocarbon contaminated site: Proceedings of SAGEEP, Arlington, Virginia, USA, 607-616, Environmental and Engineering Geophysical Society.

Delgado-Rodríguez, O., Shevnin, V., Ochoa-Valdés, J., Ryjov, A., 2006, Geoelectrical characterization of a site with hydrocarbon contamination caused by pipeline leakage: Geofísica Internacional, $45,63-72$. 
Environmental Protection Agency (EPA), 2000. Innovations in Site Characterization: Geophysical Investigation at Hazardous Waste Sites (en línea), publicado en agosto de 2000, disponible en <www. epa.gov/tio cluin.org>, consultado en enero 2013.

Hernández Márquez, J.O., Rodríguez Villuendas, A., 2008, Aplicación de métodos geoeléctricos para el diagnóstico de un sitio urbano contaminado por hidrocarburos: Facultad de Ingeniería, UNAM, Tesis de licenciatura, $149 \mathrm{p}$.

Instituto Nacional de Estadística, Geografía e Informática (INEGI), 2004, Cuaderno Estadístico Municipal de Puebla, Puebla (en línea): INEGI, publicado en 2004, disponible en <www.inegi.gob.mx>, consultado en enero del 2012.

Iris Instruments, 2012, disponible en <http://www.iris-instruments.com/ Pdf\%20file/SyscalPro_Gb.pdf>, consultado en marzo 2012.

Loke, M.H., Barker, R.D., 1996, Rapid least-squares inversion of apparent resistivity pseudosections by a quasi-Newton method: Geophysical Prospecting, 44, 131-152.

Lopes de Castro, D., Gomes Castelo Branco, R.M., 2003, 4-D ground penetrating radar monitoring of a hydrocarbon leakage site in Fortaleza (Brazil) during its remediation process: a case history: Journal of Applied Geophysics, 54, 127- 144.

Madrigal, I., 1998, Alternativas de tratamiento para la rehabilitación de sitios contaminados con hidrocarburos en México: México D.F., Posgrado en Ingeniería, Universidad Nacional Autónoma de México, Tesis de maestría, $206 \mathrm{p}$.

Orellana, E., 1982, Propección geoeléctrica en corriente continua: Madrid, España, Editorial Paraninfo, 578 p.

Pellerin, L., 2002, Applications of electrical and electromagnetic methods for environmental and geotechnical investigations: Surveys in Geophysics, 23, 101-132.

Rocha, M., 2000, Evaluación de la contaminación, criterios y alternativas de rehabilitación en los talleres de ferrocarriles nacionales de México (FNM) en Aguascalientes, Ags., México: Universidad Nacional Autónoma de México, Facultad de Ingeniería, tesis de maestría, 143 p.
Sauck, W.A., 2000, A model for the resistivity structure of LNAPL plumes and their environs in sandy sediments: Journal of Applied Geophysics, 44, 151-165.

Saval, S., Lesser, J.M., 1999, Intemperismo, efectos de hidrocarburos en suelos y acuíferos: Revista Teorema, 23, 34-35.

Secretaría de Medio Ambiente y Recursos Naturales (SEMARNAT), 2007, Norma Oficial Mexicana que establece los criterios para determinar las concentraciones de remediación de suelos contaminados por arsénico, bario, berilio, cadmio, cromo hexavalente, mercurio, níquel, plata, plomo, selenio, talio y/o vanadio (NOM-147SEMARNAT/SSA1): México, D.F., Diario Oficial de la Federación, 2 de marzo de 2007, 86 p.

Secretaría de Medio Ambiente y Recursos Naturales (SEMARNAT), 2003, Norma Oficial Mexicana que establece los límites máximos permisibles de hidrocarburos en suelos y las especificaciones para su caracterización y remediación (NOM-138-SEMARNAT/SS2003). México, D.F. Diario Oficial de la Federación, 29 de marzo de $2005,21 \mathrm{p}$.

Servicio Geológico Mexicano, 2010, disponible en <http://www.sgm.gob. $\mathrm{mx}>$, consultado en 2010.

Shevnin V., Delgado-Rodríguez, O., Fernández-Linares, L., ZegarraMartínez, H., Mousatov, A., Ryjov, A., 2005, Geoelectrical characterization of an oil-contaminated site in Tabasco, México: Geofísica Internacional, 44, 251-263.

Volkov, S., Gorbunov, A., Shevnin, V., 2000, Electrical properties of oil-polluted grounds laboratory measurements (resumen), en Proceedings for the EEGS-ES 2000 Annual Meeting: Bochum, Alemania

Manuscrito recibido: Enero 25, 2013.

Manuscrito corregido recibido: Abril 2, 2013.

Manuscrito aceptado: Abril 5, 2013. 